\title{
QUALIDADE DE PLANTAS MEDICINAIS COMERCIALIZADAS POR RAIZEIROS DE DOURADOS - MATO GROSSO DO SUL
}

\section{QUALITY OF MEDICINAL PLANTS COMMERCIALIZED BY HERB SELLERS OPERATING FROM DOURADOS - MATO GROSSO DO SUL}

\author{
Gisele de Carvalho Viana ${ }^{1}$ \\ Acadêmica de Farmácia/ Graduação. Centro Universitário da Grande Dourados \\ (UNIGRAN), Dourados, MS, Brasil. \\ https://orcid.org/0000-0003-4022-4124 \\ gisele cv1@hotmail.com \\ Renata Bordão \\ Acadêmica de Farmácia/ Graduação. Centro Universitário da Grande Dourados \\ (UNIGRAN), Dourados, MS, Brasil. \\ https://orcid.org/0000-0002-6277-594X \\ adri mestriner@hotmail.com \\ Profa. Orientadora Adriana M. Mestriner Felipe de Melo ${ }^{32}$ \\ Pesquisadora (Doutora). Centro Universitário da Grande Dourados (UNIGRAN), Dourados, \\ Mato Grosso do Sul, Brasil. \\ https://orcid.org/0000-0001-9643-7036 \\ mestriner@unigran.br \\ Prof. Coorientadora Karimi Sater Gebara ${ }^{43}$ \\ Pesquisadora Colaboradora (Doutora). Centro Universitário da Grande Dourados \\ (UNIGRAN), Dourados, MS, Brasil. \\ https://orcid.org/0000-0002-0860-3047 \\ karimi.gebara@unigran.br
}

\footnotetext{
${ }^{1}$ Participação na coleta de informações, tabulação dos dados, escrita da versão inicial do trabalho.

2 Participação ativa na construção do trabalho, discussão dos resultados e construção da versão final do trabalho. Escrita. Revisão e edição da versão final do trabalho.

${ }^{3}$ Participação ativa na construção do trabalho, discussão dos resultados e construção da versão final do trabalho. Escrita. Revisão e edição da versão final do trabalho.
}

\section{RESUMO}

A demanda por produtos à base de plantas medicinais tem aumentado fazendo-se necessário investigar sua qualidade. O objetivo desta pesquisa foi detectar a qualidade microbiológica e físico-química de Rhamnus purshiana (cáscara sagrada), Senna alexandrina (sene) e Stryphnodendrom adstrigens (barbatimão) comercializadas por raizeiros de Dourados - MS. Foram adquiridas duas amostras de cada planta de três raizeiros diferentes. Após a coleta, as amostras foram avaliadas de acordo com 
a RDC no 10 de 2010 e pela Farmacopeia Brasileira de 1988. Os valores de umidade da cáscara sagrada, do barbatimão e do sene estavam dentro dos padrões estabelecidos pela legislação vigente para droga vegetal. Somente o sene comercializado por um raizeiro foi reprovado no percentual de sujidades. $\mathrm{Na}$ análise de cinzas, uma amostra de cáscara sagrada e todas as amostras do barbatimão foram reprovados. Dentre as características microbiológicas, todas as amostras pesquisadas estavam dentro dos parâmetros exigidos pela legislação para Staphylococcus sp. Entretanto apenas 2 plantas foram aprovadas para ausência de coliformes. Os resultados da análise microbiológica das plantas medicinais evidenciam que 7 amostras foram consideradas impróprias para consumo humano. A maioria das drogas vegetais pesquisadas foram reprovadas nas análises de conteúdo informacional da embalagem, microbiologia e cinzas, sendo assim, não estavam dentro dos padrões de qualidade exigidos pela RDC no 10 , de 9 de março de 2010.

Palavras-chave: Rhamnus purshiana. Senna alexandrina. Stryphnodendrom adstrigens.

\section{ABSTRACT}

The demand for products based on medicinal plants has increased each year and as a result of the approval of the Ministério da Saúde of drugs plants is necessary to investigate its quality. The objective was to detect microbiological and physical-chemical of Rhamnus purshiana (cáscara sagrada), Senna alexandrina (sene) and Stryphnodendrom adstrigens (barbatimão) commercialized by the herb sellers in the city of Dourados, Brazil. It was acquired two samples of each plant and from three different herb sellers. After collection, the samples were evaluated according to RDC 10 of 2010 and to Pharmacopeia Brasileira (1988). The values of humidity of the plants bearberry, alumbark and senna were within the patterns. Only the Senna alexandrina commercialized by one herb seller was disapproved in the percentage of foreign matter. In the analysis of aches, only one sample of Rhamnus purshiana and all the samples of Stryphnodendrom adstrigens were disapproved. Among the microbiological characteristics, all the investigated samples were within the parameters required by law for Staphylococcus sp. However, 2 plants have been approved for the absence of coliforms. The results of microbiological testing of medicinal plants showed that 7 samples were found improper for human consumption. Most drugs plants surveyed were rejected in the analysis of information content of packaging, microbiology and ashes, so weren't within the quality standards required by the RDC No. 10, March 9, 2010.

Keywords: Rhamnus purshiana. Senna alexandrina. Stryphnodendrom adstrigens.

\section{INTRODUÇÃO}

Os produtos naturais, de forma geral, têm sido utilizados para a prevenção, tratamento e cura de enfermidades desde a antiguidade. De qualquer forma, sabe-se que a utilização das plantas medicinais na prática nutricional tem sido um aliado importante na promoção da saúde da população, atualmente denominados de nutracêuticos (CORNARA et al., 2017).

Contudo, a ingestão dos produtos naturais com finalidades nutracêuticas precisa estar associada a condições higiênico-sanitárias satisfatórias, caso contrário poderia configurar risco a saúde humana (MARTINEZ, 2013). Nesse 
sentido, a Agência Nacional de Vigilância Sanitária (ANVISA) considerando a necessidade de contribuir para a regulamentação da produção, distribuição e do uso racional de plantas medicinais, particularmente sob a forma de drogas vegetais, de modo a garantir e promover a segurança, a eficácia e a qualidade no acesso a esses produtos, adotou e publicou a RDC n 10 de 2010 (BRASIL, 2010a).

Os produtos comercializados por raizeiros deveriam respeitar os critérios de adequação à saúde e atender ao binômio segurança e eficácia, como é exigido para os produtos das demais práticas terapêuticas (SANTOS et al., 2011). Entretanto, o cumprimento as normativas nem sempre acontece. Considerando a grande demanda por produtos naturais, consequência do significativo aumento do interesse do público brasileiro por "terapias naturais", faz-se necessário investigar como esses produtos estão sendo oferecidos ao consumidor, de acordo com a legislação específica. Três espécies têm sido amplamente utilizadas como recurso terapêutico por grande parte da população brasileira e que são alvo deste trabalho, são elas: Rhamnus purshiana, Senna alexandrina e Stryphnodendrom adstrigens.

Rhamnus purshiana, conhecida popularmente como cáscara sagrada, tem sua casca preparada por decocção. Deve ser administrada por via oral de $1 / 2$ a 1 xícara de chá, antes de dormir. O uso contínuo (mais de uma semana) pode promover diarreia, perda de eletrólitos e dependência (BRASIL, 2010b).

Senna alexandrina, conhecida vulgarmente no Brasil por sene, tem como parte utilizada o fruto e os folíolos. Deve ser administrada oralmente uma xícara de chá, antes de dormir. A forma de preparo é por decocção. O uso crônico (mais de uma semana) pode promover diarreia e perda de eletrólitos (BRASIL, 2010b).

Stryphnodendrom adstrigens é conhecida popularmente por barbatimão, tem sua casca preparada por decocção. Aplicam-se compressas no local afetado 2 ou 3 vezes ao dia em lesões. Não deve ser utilizado em lesões com processo inflamatório intenso (BRASIL, 2010b).

Tendo em vista a importância do uso de plantas medicinais como complemento nutricional, o presente trabalho teve como objetivo avaliar a qualidade microbiológica e físico-química dessas drogas vegetais.

\section{MATERIAL E MÉTODOS}

As plantas escolhidas para a pesquisa foram: cáscara sagrada (Rhamnus purshiana), sene (Senna alexandrina) e barbatimão (Stryphnodendrom adstrigens). Essas plantas foram coletadas em três raizeiros da cidade de Dourados-MS (denominados 
A, $B$ e C). Essas espécies foram escolhidas por estarem contempladas no Anexo I da Resolução no 10, de 9 de março de 2010 da ANVISA. Foram adquiridas duas amostras de cada planta e de cada local. As análises foram realizadas conduzidas no Laboratório de Microbiologia da UNIGRAN em triplicata.

Foram realizadas as análises de umidade e cinzas totais, a presença macroscópica de sujidades e o conteúdo instrutivo da embalagem e do folheto informativo, conforme metodologias da Comissão Permanente de Revisão da Farmacopeia (1988).

Foram realizadas as análises microbiológicas de contagem padrão em placa, de bolores e leveduras, de Staphylococcus spp, de enterobactérias, de Salmonella e Shigella e de coliformes. As amostras sofreram diluições seriadas até $10^{-5}$. No primeiro tubo foi transferido $1 \mathrm{ml}$ da amostra para $9 \mathrm{ml}$ de líquido de diluição 1:10 (tubo 1). Este tubo foi agitado e dele transferido $1 \mathrm{ml}$ para outro tubo contendo $9 \mathrm{ml}$ de líquido de diluição 1:1.00 (tubo 2). A seguir, realizou-se nova diluição, sendo transferido $1 \mathrm{ml}$ do tubo 2 para o tubo 3 contendo $9 \mathrm{ml}$ de líquido de diluição 1: 1000. E assim por diante até o tubo 5. Após realizadas as diluições, foram semeadas $1 \mathrm{ml}$ das mesmas, em placas de Petri, no Ágar Batata (pesquisa de bolores e leveduras), no Ágar Manitol (Staphylococcus spp), no Ágar MacConkey (enterobactérias), no Ágar Salmonella e Shigella (bactérias Salmonella e Shigella) e no Ágar Verde Brilhante (coliformes).

\section{RESULTADOS E DISCUSSÃO}

$\mathrm{Na}$ análise de cinzas totais das plantas medicinais pesquisadas (Tabela 1) apenas uma amostra de cáscara sagrada e as amostras do barbatimão de todos os raizeiros estavam acima do limite permitido.

Tabela 1 - Determinação de cinzas, umidade e sujidade das amostras de plantas comercializadas em Dourados

\begin{tabular}{lcccc}
\hline Amostras & Raizeiros & Cinzas (\%) & Umidade (\%) & Sujidade (\%) \\
\hline Sene & A & 11,03 & 9,03 & 1,93 \\
& B & 10,83 & 9,07 & 1,59 \\
Cáscara sagrada & C & 8,02 & 9,24 & 1,16 \\
& A & 5,16 & 9,10 & 0,23 \\
Barbatimão & B & 5,05 & 9,11 & 0,24 \\
& C & 9,01 & $9,89^{*}$ & 0,30 \\
& A & $5,30^{*}$ & 9,99 & 0,45 \\
& B & $2,69^{*}$ & 11,26 & 0,04 \\
& C & $4,54^{*}$ & 11,47 & 0,00
\end{tabular}

* resultados acima dos valores de referência. Valores de referência da Comissão Permanente de Revisão da Farmacopeia (1988): cinzas: até $2 \%$ para barbatimão, até $6 \%$ para cáscara sagrada, até
$12 \%$ para sene; umidade: até $15 \%$ para barbatimão, até $12 \%$ para cáscara sagrada e para sene; sujidades: até $2 \%$ para barbatimão, até $1 \%$ para cáscara sagrada, até $5 \%$ para sene. 
Considerando a Comissão Permanente de Revisão da Farmacopeia (2003) o barbatimão deve ter no máximo $2 \%$, de acordo com a Comissão Permanente de Revisão da Farmacopeia (1996) a cáscara sagrada no máximo $6 \%$ e o sene, no máximo, $12 \%$. As cinzas totais incluem as derivadas de tecido vegetal (cinzas fisiológicas) e de materiais estranhos, especialmente areia e terra aderente à superfície da droga (cinzas não fisiológicas) (COMISSÃO PERMANENTE DE REVISÃO DA FARMACOPÉIA, 1988).

Sendo assim, as amostras do barbatimão apresentaram contaminantes inorgânicos não voláteis.

Todas as amostras pesquisadas estavam de acordo com as especificações contidas na RDC № 10 de 2010 quanto à umidade e sujidades. Considerando que os valores máximos de umidade são 15\% para barbatimão e $12 \%$ para cáscara sagrada e para o sene (COMISSÃO DA FARMACOPEIA PORTUGUESA, 2002).

O teor de sujidade para os folíolos do sene deve ser no máximo $5 \%$, a cáscara sagrada no máximo $1 \%$ e 0 barbatimão, no máximo, $2 \%$. Isso porque as drogas vegetais devem estar, o quanto possível, isentas de fungos, insetos e outros materiais contaminantes, conforme a Comissão Permanente de Revisão da Farmacopeia (1988).
Segundo Melo et al. (2004), entretanto, o excesso de elementos estranhos é um problema frequente dos produtos à base de plantas medicinais comercializadas no Brasil, pois, constataram que todas as amostras de boldo (Peumus boldus Molina) e metade das amostras de pata-de-vaca (Bauhinia spp.) possuíam excesso de constituintes indesejáveis. A grande quantidade de impurezas pode estar relacionada a um processo inadequado de separação das partes vegetais e/ou a limpeza precária na produção (MELO et al., 2007).

Dentre as características
microbiológicas, todas as amostras
pesquisadas estavam dentro dos
parâmetros exigidos pela legislação para
Staphylococcus spp. Entretanto, pôde-se evidenciar a contagem de Salmonella e Shigella associada à contagem elevada de bolores e leveduras, que ocorreu em 5 e em três amostras analisadas, respectivamente. Já na análise de coliformes apenas duas plantas foram aprovadas. Os resultados da análise microbiológica das plantas medicinais, contidos na Tabela 2, evidenciam que sete amostras foram consideradas impróprias para consumo humano. 
Tabela 2 - Resultados das análises microbiológicas das plantas medicinais comercializadas por 3 raizeiros de Dourados.

\begin{tabular}{|c|c|c|c|c|c|c|c|}
\hline Amostras & LOCAL & $\begin{array}{l}\text { Bolores } \\
\text { leveduras } \\
(\mathrm{UFC} / \mathrm{g})\end{array}$ & e & $\begin{array}{l}\text { S. aureus } \\
\text { (UFC/g) }\end{array}$ & $\begin{array}{l}\text { Coliformes } \\
\text { totais } \\
(\mathrm{UFC} / \mathrm{g})\end{array}$ & $\begin{array}{l}\text { Salmonella } \\
\text { Shigella } \\
\text { (UFC/g) }\end{array}$ & $\begin{array}{l}\text { Coliformes } \\
\text { fecais } \\
\text { (UFC/g) }\end{array}$ \\
\hline \multirow[t]{3}{*}{ Sene } & $A$ & $<10^{3}$ & & $<10^{5}$ & a & $\mathrm{a}$ & $<10^{2^{*}}$ \\
\hline & $B$ & $<10^{5^{*}}$ & & $<10^{5}$ & $<10^{2}$ & $<10^{2^{*}}$ & $<10^{3^{*}}$ \\
\hline & $\mathrm{C}$ & $<10^{5^{*}}$ & & $<10^{4}$ & $<10^{3}$ & $<10^{3^{*}}$ & $<10^{4^{*}}$ \\
\hline \multirow{3}{*}{$\begin{array}{l}\text { Cáscara } \\
\text { sagrada }\end{array}$} & $A$ & $<10^{2}$ & & 10 & a & a & $<10^{2^{*}}$ \\
\hline & B & $<10^{2}$ & & $<10^{2}$ & $<10^{2}$ & a & a \\
\hline & C & $<10^{3}$ & & $<10^{3}$ & a & a & a \\
\hline \multirow[t]{3}{*}{ Barbatimão } & $A$ & $<10^{3}$ & & $<10^{3}$ & $<10^{3}$ & $10^{*}$ & $<10^{2^{*}}$ \\
\hline & B & $<10^{2}$ & & $<10^{3}$ & $<10^{5^{*}}$ & $<10^{3^{*}}$ & $<10^{2^{*}}$ \\
\hline & C & $<10^{5^{*}}$ & & $<10^{2}$ & $<10^{3}$ & $<10^{2^{*}}$ & $10^{*}$ \\
\hline ** & & $10^{4}$ & & $10^{5}$ & $<10^{4}$ & a & a \\
\hline
\end{tabular}

* resultados acima dos valores de referência expressos em UFC/g.

** Valores de referência da RDC 10 de 9 de março de 2010;

a: ausência

Apenas na embalagem de sene do

A ausência de informação, por muitas vezes, pode provocar enganos em sua administração e manejo e assim possuir efeito placebo ou causar malefícios ao invés de benefícios para a saúde do consumidor. As embalagens das plantas deveriam ser recipientes bem fechados e deveriam ser armazenados protegidos da luz; e no caso do barbatimão protegido do calor também (COMISSÃO PERMANENTE DE REVISÃO DA FARMACOPEIA BRASILEIRA, 2003; COMISSÃO PERMANENTE DE REVISÃO DA FARMACOPEIA BRASILEIRA, 1996). Não foi observada nenhuma dessas medidas, pois as embalagens ficavam expostas a luz e ao calor diariamente e uma embalagem de barbatimão do raizeiro $B$ não estava bem fechado.

Segundo Brasil (2010b), nenhuma das 3 plantas é utilizada por infusão e na embalagem de sene do raizeiro $A$ tinha essa informação errônea do modo de preparo e a posologia estava errada. raizeiro $A$ tinha a informação de que deve ser preparada a decocção imediatamente antes do uso. Sendo que apenas o barbatimão dispensaria essa informação, pois pode ser preparada para mais de uma dose já que é utilizada de 2 a 3 vezes por dia (BRASIL, 2010a).

A embalagem do barbatimão do raizeiro $A$ errou ao apontar o seu uso por via oral (sendo que é tópico) e o modo de preparo não era a mesma concentração do estabelecido e posologia pelo Anexo I da RDC no 10, de 9 de março de 2010. A embalagem do sene do raizeiro $B$ estava sem modo de preparo e posologia errada.

Um dos raizeiros adquire as suas plantas de um fornecedor de outro estado e os outros dois raizeiros adquirem de uma pessoa que compra a matéria-prima, embala e rotula da cidade de DouradosMS.

Apenas a cáscara sagrada do raizeiro $A$ e todas as plantas do raizeiro $C$ apresentaram o nome científico da planta na embalagem. $O$ sene do raizeiro $A$ e $B$ 
apresentavam na embalagem a parte utilizada da droga vegetal. A ausência dos nomes científicos e da parte da planta utilizada nos produtos abre uma brecha para a adulteração e favorece a introdução de outras partes vegetais que não contenham o princípio ativo desejado ou o possuam em baixa quantidade (MELO et al., 2007).

A ausência ou apresentação de informações obrigatórias de maneira incompleta demonstra a necessidade de padronização e inserção de informações essenciais a fim de possibilitar o uso consciente e correto pelo consumidor (MELO et al., 2007).

As plantas adquiridas do raizeiro $A$ continham aproximadamente $26 \%$ das informações obrigatórias, do raizeiro B aproximadamente $9 \%$ e do raizeiro C $30 \%$.

Quanto ao controle de qualidade as amostras de barbatimão apresentaram limites acima dos previstos de cinzas totais em todas as amostras analisadas (tabela 1). De forma curiosa, os agentes microbianos também foram encontrados em maior proporção nas amostras dos diferentes locais analisados (tabela 2).

Quanto às indicações terapêuticas atribuídas aos produtos verificou-se que nem todos possuem comprovação científica que suportem as propriedades biológicas sugeridas e que, em alguns produtos, não consta indicação alguma (Quadro 1).

Quadro 1 - Indicações transcritas literalmente das embalagens das plantas medicinais.

\begin{tabular}{|c|c|c|}
\hline AMOSTRAS & $\begin{array}{l}\text { Indicação atribuída no rótulo } \\
\text { analisado }\end{array}$ & $\begin{array}{l}\text { Indicação do Anexo I da RDC no } 10 \\
\text { de } 09 \text { de março de } 2010\end{array}$ \\
\hline Barbatimão A & $\begin{array}{lcr}\text { Úlcera, } & \text { impigen, } & \text { hérnias, } \\
\text { licorreia, } & \text { tônico, } & \text { blenorragia, } \\
\text { hemorragia } & \text { uterina, } & \text { catarro } \\
\text { vaqinal, diarreia e desenteria" } & \end{array}$ & $\begin{array}{l}\text { Cicatrizante de lesões e antisséptico } \\
\text { tópico na pele e mucosas bucal e } \\
\text { genital }\end{array}$ \\
\hline Sene A & $\begin{array}{l}\text { "Possui propriedades purgativas, } \\
\text { laxativas, febrífugas e ajuda a eliminar } \\
\text { manchas do corpo }\end{array}$ & Constipação intestinal eventual \\
\hline Cascara sagrada $A$ & Não consta & Constipação intestinal eventual \\
\hline Barbatimão B & $\begin{array}{l}\text { "Sicatrizante cura feridas, ulcera do } \\
\text { estomago, gastrite, varizes inflamação" }\end{array}$ & $\begin{array}{l}\text { Cicatrizante de lesões e antisséptico } \\
\text { tópico na pele e mucosas bucal e genital }\end{array}$ \\
\hline Sene B & $\begin{array}{l}\text { Possui propriedades purgativas } \\
\text { laxativas, depurativas, febrífugas e } \\
\text { ajuda a eliminar manchas do corpo }\end{array}$ & Constipação intestinal eventual \\
\hline Cascara sagrada $B$ & $\begin{array}{l}\text { "Laxante, elimina manchas da pele, } \\
\text { acnes, depurativo do sangue, prisão de } \\
\text { ventre, emagrecedor e diurético" }\end{array}$ & Constipação intestinal eventual \\
\hline Barbatimão C & Não consta & $\begin{array}{l}\text { Cicatrizante de lesões e antisséptico } \\
\text { tópico na pele e mucosas bucal e genital }\end{array}$ \\
\hline Sene C & Não consta & Constipação intestinal eventual \\
\hline Cascara sagrada $\mathrm{C}$ & Não consta & Constipação intestinal eventual \\
\hline
\end{tabular}

\section{CONSIDERAÇÕES FINAIS}

As drogas vegetais pesquisadas foram reprovadas nas análises de 
conteúdo informacional da embalagem, microbiologia e cinzas, sendo assim, não estavam dentro dos padrões de qualidade exigidos pela ANVISA. Elas carecem de informações técnico-científicas que propiciem o seu uso correto e racional. Muitos produtores desconhecem os cuidados que se deve ter nas diversas etapas para a obtenção de matériasprimas e/ou produtos de qualidade adequada e não contam com a orientação de profissionais capacitados e assim comercializam produtos de má qualidade.

\section{REFERÊNCIAS BIBLIOGRÁFICAS}

BRASIL. Agência Nacional de Vigilância Sanitária. Resolução no 10, de 9 de março de 2010. Brasília. Disponível em: http://www.brasilsus.com.br/legislacoes/rd c/103202-10. Acesso em: 2 maio 2010a.

BRASIL. Agência Nacional de Vigilância Sanitária. Resolução no 10, de 9 de março de 2010: Anexo 1. Brasília. Disponível em:

http://www.brasilsus.com.br/images/stories lanexoportaria/anexo10rdc10.pdf. Acesso em: 2 maio 2010b.

\section{COMISSÃO DA FARMACOPEIA}

PORTUGUESA. Farmacopeia

Portuguesa. 7. ed. Lisboa, jul. 2002. 1 CD-ROM.

COMISSÃO PERMANENTE DE REVISÃO DA FARMACOPÉIA BRASILEIRA. Farmacopeia Brasileira:

Parte 1. 4. ed. São Paulo: Atheneu, 1988.

COMISSÃO PERMANENTE DE

REVISÃO DA FARMACOPÉIA

BRASILEIRA. Farmacopeia Brasileira:
Parte 2, fascículo 1. 4. ed. São Paulo: Atheneu, 1996.

\section{COMISSÃO PERMANENTE DE REVISÃO DA FARMACOPÉIA BRASILEIRA. Farmacopeia Brasileira: Parte 2, fascículo 4. 4. ed. São Paulo: Atheneu, 2003}

CORNARA, L.; et al. Therapeutic Properties of Bioactive Compounds from Different Honeybee Products. Front. Pharmacol., v.8, n. 412, 2017.

MARTINEZ, Sílvia. A nutrição e a alimentação como pilares dos programas de promoção da saúde e qualidade de vida nas organizações. Revista 0 mundo da Saúde, v. 37, n. 7, p. 201-207, 2013.

MELO, J. G. et al. Qualidade de produtos a base de plantas medicinais comercializados no Brasil: castanha-daíndia (Aesculus hippocastanum L.), capimlimão (Cymbopogon citratus (DC.) Stapf) e centela (Centella asiática (L.) Urban).

Revista Acta Botânica Brasílica, v.21, n.1, p.27-36, 2007.

MELO, J.G. et al. Avaliação da qualidade de amostras comerciais de boldo (Peumus boldus Molina), pata-de-vaca (Bauhinia spp.) e ginco (Ginkgo biloba L.). Revista Brasileira de Farmacognosia, v.14, n.2, p.111-120, 2004.

SANTOS, Ravely L. et al. Análise sobre a fitoterapia como prática integrativa no Sistema Único de Saúde. Revista Brasileira de Plantas Medicinais, v. 13, n. 4, p. 486-491, 2011. 